\title{
Review of: "CURT1A and CURT1C mediate distinct stages of plastid conversion in Arabidopsis"
}

Sheng Teng

Potential competing interests: The author(s) declared that no potential competing interests exist.

The authors observed the process of the etioplasts to chloroplast transition in Arabidopsis cotyledons after iillumination. The artifacts in fixation was avioded and the image contrast ws enhanced by using highpressure freezing scanning transmission ET. So, more detailed structure of etioplasts and the intermediate structures during the transition from prolamellar bodies (PLB) to thylakoid were obtained. The authors also studied the functions of CURVATURE THYLAKOID1 (CURT1) family proteins in PLB and the formation of thylakoid using this improved approach. They found that CURT1A works on the stack assembly. CURT1C plays a role for PLB biogenesis. Thers resutls are important for the feild of plant chloroplast development and photosynthesis.

This is a excellent cytological work but less general interesting. I thinks to it is better to show more complehansive results and discussed deeply to increase the attractiveness. 